\title{
Inflammatory Syndrome as the Initial Manifestation of Retroperitoneal Tuberculosis in a Pregnant Woman
}

\author{
Marwene Grira $^{1}$, Michel Boulvain ${ }^{2}$, Jean-Paul Janssens ${ }^{3}$ \\ ${ }^{1}$ Department of Community Medicine, Primary Care and Emergency Medicine, University Hospitals of Geneva, Switzerland \\ ${ }^{2}$ Division of Obstetrics, Department of Gynecology and Obstetrics, University Hospitals of Geneva, Switzerland \\ ${ }^{3}$ Division of Pulmonary Diseases, Department of Internal Medicine Specialties, University Hospitals of Geneva, Switzerland
}

\section{Doi: 10.12890/2018_000865 - European Journal of Case Reports in Internal Medicine - ( ) EFIM 2018}

\begin{abstract}
Received: 15/02/2018
Accepted: 08/05/2018

Published: $24 / 05 / 2018$
\end{abstract}

How to cite this article: Grira M, Boulvain M, Janssens JP. Inflammatory syndrome as the initial manifestation of retroperitoneal tubercolosis in a pregnant woman. EJCRIM 2018;5: doi:10.12890/2018_000865.

Conflicts of Interests: The Authors declare that there are no competing interests.

This article is licensed under a Commons Attribution Non-Commercial 4.0 License

\section{ABSTRACT}

A 20-year-old asylum seeker presented with vomiting and left thigh pain, with a biological inflammatory syndrome. Pregnancy was diagnosed. Investigations revealed a pseudo-cystic, 20-cm-long retroperitoneal abscess, biopsy of which confirmed the diagnosis of tuberculosis. Evolution after cyst drainage and under conventional anti-tuberculosis treatment was favourable. An abdominal location of tuberculosis is rare and its diagnosis is difficult especially in countries with a low incidence of the disease. Unexplained abdominal manifestations and/or persistent biological inflammatory syndrome, especially in high-risk groups, should raise the suspicion of tuberculosis.

\section{LEARNING POINTS}

- Abdominal tuberculosis (TB) is a challenging diagnosis especially in low-incidence countries where the disease is rarely suspected.

- In low-incidence countries, abdominal TB should be suspected in cases of unexplained abdominal manifestations and/or persistent inflammatory syndrome, especially in high-risk groups.

- The diagnosis of abdominal TB is based on a range of anamnestic and clinical symptoms and signs, imaging, culture, and invasive procedures for histology.

\section{KEYWORDS}

Abdominal tuberculosis, inflammatory syndrome, pregnancy

\section{CASE DESCRIPTION}

A 20-year-old female Eritrean asylum seeker, who had arrived in Switzerland 6 months previously, presented to our primary care medicine department. She complained of nausea, vomiting and a pain in the left thigh of 15 days' duration, without a history of trauma.

Physical examination was unremarkable except for tenderness on palpation of the left thigh. Biological tests revealed microcytic anaemia with a haemoglobin of $85 \mathrm{~g} / \mathrm{l}$ (normal $120-160 \mathrm{~g} / \mathrm{l}$ ) and an MCV of $78 \mathrm{fl}$ (normal 82-98 fl), an inflammatory syndrome with a CRP of $88 \mathrm{mg} / \mathrm{l}$ (normal 0-10 mg/l) and a sedimentation rate of $77 \mathrm{~mm} / \mathrm{h}$ (normal 0-20 mm/h), and a slight disturbance of liver function with an ALP of 159 $\mathrm{U} / \mathrm{I}$ (normal 25-102 U/I), GGT of $67 \mathrm{U} / \mathrm{I}$ (normal 9-35 U/I), and normal ASAT and ALAT. $\beta$-hCG was increased to 181,567 U/I.

Obstetrical ultrasound confirmed a 10-week pregnancy and the diagnosis of hyperemesis gravidarum was made. The woman was discharged. To investigate the inflammatory syndrome, an extensive infection screen was performed including bacterial (blood, urine and stool cultures, 
brucellosis and syphilis serologies), viral (VZV, CMV, EBV, parvovirus B19, HTLV 1 and 2, measles, rubella, viral hepatitis and HIV) and parasitic (toxoplasmosis, leishmaniasis and malaria) screens. Tests confirmed past exposure to rubella, varicella and hepatitis A. The other tests were negative. The patient was also screened for TB: IGRA (QuantiFERON ${ }^{\circledR}$-TB Gold) was positive, but chest X-ray was normal. Finally, the left thigh pain was investigated by an US scan of the soft parts of the thigh, which was normal.

Abdominal and pelvic US performed at 20 weeks of amenorrhoea revealed a large left abdominal mass suggestive of an ovarian cyst, responsible for a left inguinal hernia. An MRI scan of the pelvis confirmed a left pseudo-cystic, 20-cm-long retroperitoneal abscess which extended along the left iliopsoas muscle and was associated with various tissue injuries along the left sympathetic chain (Figs. 1-3)

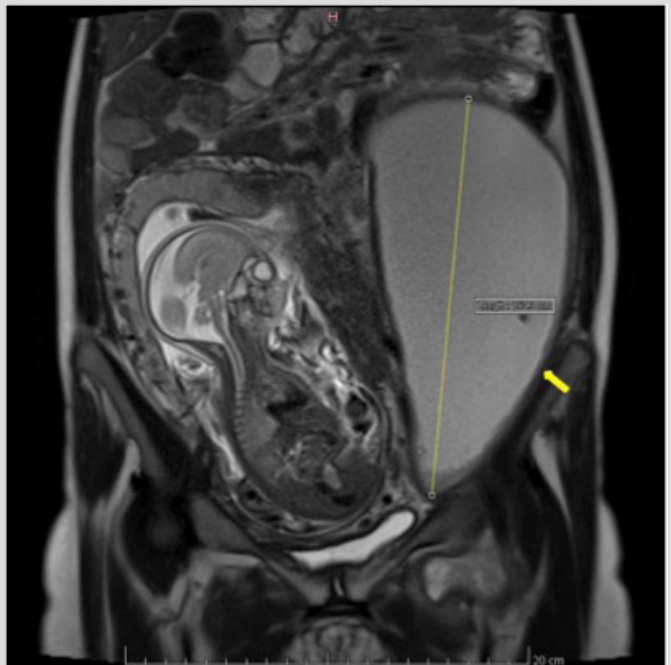

Figure 1 Sagittal section at 20 weeks of amenorrhoea showing the fetus facing the retroperitoneal mass (yellow arrow)

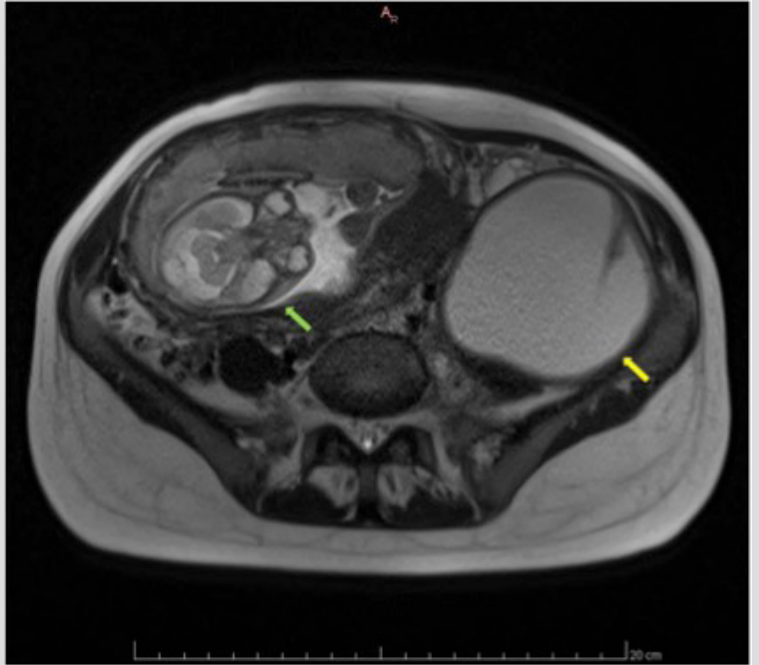

Figure 2. Cross-section at 20 weeks of amenorrhoea showing the fetus (green arrow) facing the retroperitoneal mass (yellow arrow)

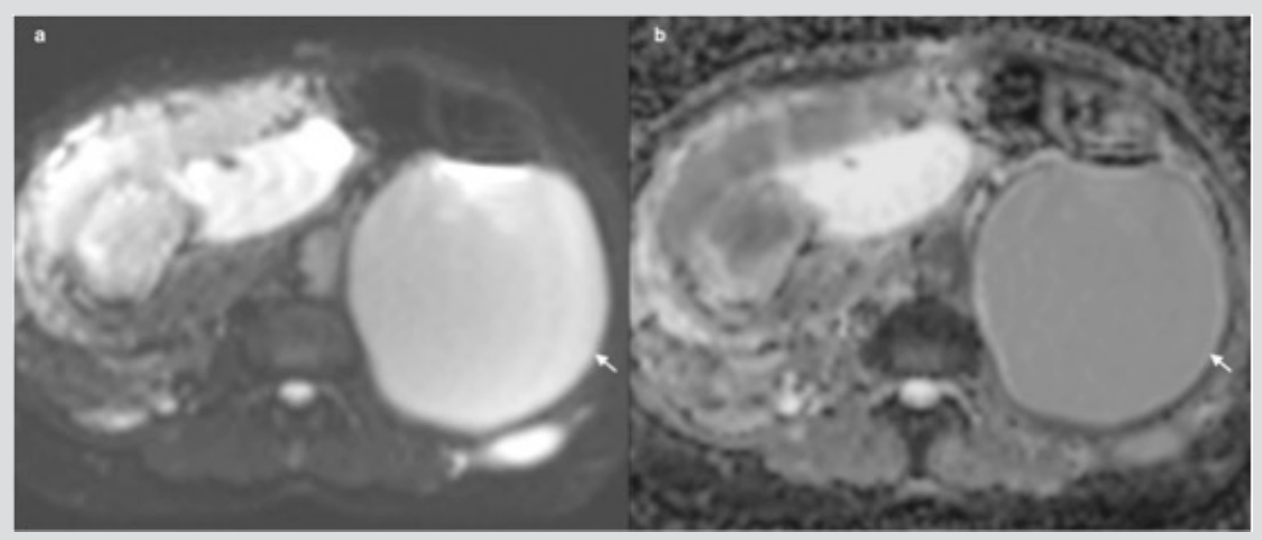

Figure 3. (a) Diffusion sequence b0 and (b) apparent diffusion coefficient (ADC) mapping. The axial plane in the upper abdomen shows $A D C$ restriction (arrow on ' 'a'), suggesting a tuberculosis collection. The denser or more cellular the biological tissue (e.g. pus), the less the diffusion of water and so the ADC. Low ADC appears in hypersignal on the diffusion sequence (a)

The radiological characteristics of the mass suggested a paraganglioma. However, a 24-hour urine and plasma fractionated metanephrines and catecholamines were normal, which excluded the diagnosis. Biopsy of the pseudo-cystic retroperitoneal abscess under US showed a granulomatous inflammation with giant cells, necrosis and acid-fast bacilli. PCR (GeneXpert) confirmed the diagnosis of TB.

A 14F catheter was inserted into the cyst and allowed drainage of about 3,000 $\mathrm{ml}$ of fluid over a few days. Conventional TB treatment (rifampicin, isoniazid, pyrazinamide and ethambutol) with fixed-dose combination tablets was started. No immunosuppressive factors or 
evidence of vertebral involvement were found. Evolution under treatment, and monthly monitoring by US, was favourable, with a transient and spontaneously reversible increase in liver tests.

Pregnancy was complicated by umbilical cord prolapse which required a caesarean section at 33 weeks of amenorrhoea. The patient gave birth to a healthy baby boy.

\section{DISCUSSION}

Abdominal TB represents about $6 \%$ of extra-pulmonary forms ${ }^{[1]}$. It can occur at any age with a peak frequency between 21 and 45 years of age $^{[2]}$. The distribution by sex varies according to studies. A female predominance is observed in endemic countries, while a male predominance is reported in many European countries. This is explained by the larger percentage of males among immigrants, in whom abdominal TB is more frequent than among the local population ${ }^{[3]}$. The clinical manifestations of abdominal TB are extremely varied, which often results in a misleading picture and delayed diagnosis. Consequently, additional examinations are very important for correct diagnosis ${ }^{[2]}$.

Peritoneal TB is the most frequent form of abdominal TB ${ }^{[1]}$ which can also affect the gastro-intestinal tract, mesentery, abdominal lymph nodes, liver, spleen and pancreas ${ }^{[4]}$. However, there is no consensus definition of abdominal TB. TB of the genital and urinary systems, which is anatomically considered abdominal TB, is usually excluded from studies and guidelines for abdominal TB. This situation makes comparison between data from different studies difficult ${ }^{[5]}$.

The treatment of abdominal TB is based on the same protocol as that for pulmonary TB with 2 months of quadritherapy, followed by 4 months of dual therapy ${ }^{[6]}$. Global mortality varies according to studies, ranging from $3 \%$ to $30 \%$ [2,7,8].

Pregnancy does not adversely impact the course of TB infection. However, it may mimic and mask the symptoms of early TB and thus delay its diagnosis ${ }^{[9]}$. Non-treated TB in the pregnant woman may lead to congenital TB in the infant. Comparative studies from India, Mexico and Taiwan found that infants born to mothers with TB have a two- to three-fold increased risk of neonatal complications such as prematurity and fetal growth retardation, as well as a six-fold increase in perinatal mortality ${ }^{[10-12]}$. The risk of transmission of TB to the infant is greater than the risks associated with TB treatment administered to the mother ${ }^{[9]}$. Therefore, treatment should be started whenever the probability of maternal disease is moderate to high. The treatment of a pregnant woman is the same as that of a non-pregnant woman ${ }^{[13]}$. Antituberculosis drugs do not have teratogenic effects in humans, even though they cross the placenta ${ }^{[13]}$.

Many factors made the diagnosi s in this case challenging:

- Constitutional symptoms (fever, night sweats, fatigue and weight loss) were absent or hidden by the context of pregnancy (e.g. weight gain).

- There were no digestive symptoms, especially no abdominal pain, which is a usual symptom in $74-94 \%$ of patient ${ }^{[7,14]}$ with abdominal TB.

- The main symptom on which we based our clinical reasoning was the inflammatory syndrome which is a frequent and non-specific sign.

- The patient had misleading symptoms such as the thigh pain due to the inguinal hernia or the hyperemesis gravidarum, which initially diverted the diagnosis.

\section{REFERENCES}

1. Mazza-Stalder J, Nicod L, Janssens JP. La tuberculose extrapulmonaire. Rev Mal Respir 2012;29:566-578.

2. Abdallah M, Larbi T, Hamzaoui S, Mezlini E, Harmel A, Ennafaa M, et al. Tuberculose abdominale: étude rétrospective de 90 cas. Rev Med Interne 2011;32:212-217.

3. Thoreau N, Fain O, Babinet P, Lortholary O, Robineau M, Valeyre D, et al. Tuberculose péritonéale: 27 cas dans la banlieue nord-est de Paris. Int J Tuberc Lung Dis 2002;6:253258.

4. Uygur-Bayramicli O, Dabak G, Dabak R. A clinical dilemma: abdominal tuberculosis. World J Gastroenterol 2003;9:1098-1101.

5. Fillion A, Ortega-Deballon P, Al-Samman S, Briault A, Brigand C, Deguelte S, et al. Abdominal tuberculosis in a low prevalence country. Med Mal Infect 2016;46:140-145.

6. Blumberg HM, Burman WJ, Chaisson RE, Daley CL, Etkind SC, Friedman LN, et al. American Thoracic Society/Centers for Disease Control and Prevention/Infectious Diseases Society of America: treatment of tuberculosis. Am J Respir Crit Care Med 2003;167:603-662.

7. Chou CH, Ho MW, Ho CM, Lin PC, Weng CY, Chen TC, et al. Abdominal tuberculosis in adult: 10-year experience in a teaching hospital in central Taiwan. J Microbiol Immunol Infect 2010;43:395-400.

8. Tan KK, Chen K, Sim R. The spectrum of abdominal tuberculosis in a developed country: a single institution's experience over 7 years. J Gastrointest Surg 2009;13:142-147.

9. Gould JM, Aronoff SC. Tuberculosis and pregnancy-maternal, fetal, and neonatal considerations. Microbiol Spectr 2016;4. doi:10.1128/microbiolspec.TNMI7-0016-2016.

10. Jana N, Vasishta K, Jindal SK, Khunnu B, Ghosh K. Perinatal outcome in pregnancies complicated by pulmonary tuberculosis. Int J Gynaecol Obstet 1994;44:119-124.

11. Figueroa-Damian R, Arredondo-Garcia JL. Neonatal outcome of children born to women with tuberculosis. Arch Med Res 2001;32:66-69.

12. Lin HC, Lin HC, Chen SF. Increased risk of low birthweight and small for gestational age infants among women with tuberculosis. BJOG 2010;117:585-590.

13. Nahid P, Dorman SE, Alipanah N, Barry PM, Brozek JL, Cattamanchi A, et al. Official American Thoracic Society/Centers for Disease Control and Prevention/Infectious Diseases Society of America clinical practice guidelines: treatment of drug-susceptible tuberculosis. Clin Infect Dis 2016;63:e147-e195.

14. Amouri A, Boudabbous M, Mnif L, Tahri N. Profil actuel de la tuberculose péritonéale: étude d'une série tunisienne de 42 cas et revue de la littérature. Rev Med Interne 2009;30:215-220. 